\title{
Zhongliang Shi
}

\section{Die Zukunft der Staatsunternehmen in China}

Vorbemerkung der Redaktion: Der folgende Beitrag ist Teil eines in China erschienenen Buches, das sich umfassend mit der Restrukturierung des Unternehmenssektors befasst. Der Verfasser ist Präsident der Jiangxi University of Finance and Economics, die zu den sechs Universitäten des Finanzministeriums in Peking gehört. Der Autor ist an der Formulierung der Reform der chinesischen Staatsunternehmen direkt beteiligt, er ist stellvertretender Vorsitzender der „Chinese Institution for Promoting Industrial and Economic Research \& Development“, geschäftsführendes Mitglied des "Jiangxi Provincial Consultancy and Decision-Making Committee“ und stellvertretender Vorsitzender der „Jiangxi Industrial Economic League“. Die Ausführungen von Zhongliang Shi dokumentieren die „offizielle“ Sichtweise, die Erwartungen und Absichten, mit der von Seiten der KP und der Regierung an die Restrukturierung des Unternehmenssektors herangegangen wird. Eine kritische Analyse dieser Restrukturierung liefert der Aufsatz von Raymond Lau.

\section{Die Gesamtkonzeption der Umstrukturierung des staatlichen Unternehmenssektors}

Mit der Umstrukturierung der staatseigenen Unternehmen (SEU) wird angestrebt, die Rolle der Staatswirtschaft zu reduzieren. Nur noch große Unternehmen und Unternehmensgruppen sollen weiterhin vom Staat geführt werden, kleinere Unternehmen sind freizugeben. Die Vermögensstruktur der Großunternehmen soll durch Umwandlung in Aktiengesellschaften, durch Fusionen, aber auch durch Konkurse, effizienter werden. Die Gesamtkonzeption der strategischen Umstrukturierung der SEU umfaßt drei Ebenen, die nun im einzelnen behandelt werden.

\subsection{Rückzug der staatlichen Unternehmen auf wenige Branchen}

Das Grundprinzip der Restrukturierung des SEU besteht in der Konzentration des staatlichen Vermögens auf große, effiziente und konkurrenzfähige Unternehmen, die vor allem in Bereichen von strategischer Bedeutung tätig sind. Daraus ergeben sich die folgenden Prioritäten für die Staatswirtschaft:

- Bereiche, die für die Staatssicherheit wichtig sind, wie Rüstungsproduktion, Raumfahrt und Münzwesen.

- Große Infrastrukturprojekte wie die Regulierung von Flüssen, groß angelegte Projekte zur Erschließung von nicht reproduzierbaren Ressourcen (wie Erdöl, Erdgas und Kohlebergwerke). Die nichtstaatlichen Sektoren sind heutzutage noch nicht in der Lage, diese Projekte zu übernehmen, bzw. wollen 
dies nicht. Internationale Finanzgruppen sind zwar daran interessiert, sich z.B. in den Bereichen Energie, Grundstoffe, Verkehr, Telekommunikation etc. $\mathrm{zu}$ engagieren und langfristige Investitionen zu tätigen, aber der Staat muss sich hier eine führende Rolle sichern.

- Handelsunternehmen und Banken mit besonderer Bedeutung. Die Staatswirtschaft muss die Kontrolle über nationale oder provinzübergreifende regionale Handelsunternehmen behalten, damit die Stabilität des Angebots und der Preise gewährleistet wird. Durch die Kontrolle des Bankensystems sowie anderer Finanzinstitute kann der Staat die Manipulation und Kontrolle des Finanzsystems seitens kleiner Gruppen oder privater Personen verhindern, um somit das gesellschaftliche Interesse zu schützen und einer Finanzkrise vorzubeugen.

- Schlüsselindustrien wie Maschinenbau, elektrotechnische Industrie, Petrochemie, Automobilbau, Bauindustrie, Leichtindustrie, Textilindustrie und Tabakindustrie. Dadurch, dass die Staatswirtschaft in diesen Branchen eine führende Rolle spielt, sollen vor allem folgende Ziele erreicht werden: steigende Skalenerträge, größere Wertschöpfung und größere Marktanteile, erhöhte internationale Wettbewerbsfähigkeit, gesicherte fiskalische Einnahmen. Außerdem kann die Staatswirtschaft mittels dieser Sektoren die Rolle als Lokomotive und Lenker der gesellschaftlichen Investitionen übernehmen.

- Technologieentwicklung. Die Entwicklung von Hochtechnologien ist für Ausbildung und Forschung sowie die langfristige Entwicklung des Landes von zentraler Bedeutung. Sie stellt auch die Basis für den Aufbau einer modernen Verteidigung dar. Der Staat soll der Forschung und Entwicklung in diesem Bereich fiskalische Unterstützung zukommen lassen. Zudem soll er diese Branche durch Investitionsregulierung fördern.

Schließlich sollten noch zwei Aspekte beachtet werden: Erstens ist die skizzierte Setzung strategischer Prioritäten von dynamischer Natur, sie muß der Entwicklung der Volkswirtschaft und den Veränderungen im Zuge des Übergangs zur Marktwirtschaft rechtzeitig angepasst werden. Zweitens zeigen diese Prioritäten nur die grobe Richtung und das ungefähre Ausmass für den Umbau der Staatswirtschaft auf. Dabei wird der Zufluss von nichtstaatlichem Kapital in diese Bereiche nicht prinzipiell ausgeschlossen. Mit Ausnahme weniger Bereiche und unter Beibehaltung der führenden Rolle der Staatswirtschaft kann auch nichtstaatliches Kapital in den genannten Branchen investiert werden. Selbstverständlich soll der Staat, je nach der konkreten Situation und organisatorischen Besonderheit einzelner Branchen und Sektoren, den Grad der Involvierung des nichtstaatlichen Kapitals umsichtig planen.

\subsection{Die Restrukturierung der Vermögensstruktur der Staatsunternehmen}

Die folgenden Möglichkeiten der Vermögensrestrukturierung gibt es bereits:

- Durchführung von Fusionen und Akquisitionen zur Optimierung der Kapitalstruktur. Dies bewirkt ersichtliche Veränderungen im Hinblick auf die Re- 
duzierung der Verluste sowie auf die Regulierung und Optimierung der Kapitalstruktur der SEU. Die konkurrenzfähigen Unternehmen können alle Gebäude, Produktionsstätten usw. der übernommenen Unternehmen nutzen und eine kostengünstige Expansion erzielen, während die nicht konkurrenzfähigen Unternehmen von Reputation, Managementerfahrung, Technologie usw. der konkurrenzfähigen Unternehmen profitieren und sich dadurch erneuern können. Die Pilotphase der „Optimierung der Kapitalstrukturierung“ in den Städten begann im Februar 1994 mit 18 Teststädten und wurde auf 111 große und mittlere Städte ausgedehnt. Laut Statistik wurden 1996 in den Teststädten 1099 Unternehmen mit einem Konkursverfahren geschlossen, 1192 Unternehmen wurden fusioniert und umstrukturiert. Im Jahre 1997 waren 2980 SEU von Fusion und Konkurs betroffen, so dass Vermögenswerte von 415,5 Mrd. Yuan und 5,6 Mio. Beschäftige neu zugeordnet wurden.

- Durchführung von Leasing, vertragsmäßiger Übernahme der Betriebsführung und treuhänderischer Verwaltung. Über diese Methode sollen mittelgroße und kleine SEU frei gegeben und belebt werden. Starke Großunternehmen können das Betriebsvermögen anderer Unternehmen erwerben. Einerseits vermeiden sie damit die Schwierigkeiten, die mit einer einmaligen großen Investition verbunden sind, anderseits erreichen sie eine schnelle Vergrößerung ihrer Produktionskapazitäten. In den letzten Jahren ist ein neues Modell in der Praxis entstanden: die treuhänderische Verwaltung. Dieses Modell wird auch als „die effiziente Streckung der vertraglichen Übernahme der Betriebsführung“ und als „sanfter Übergang zur Fusion“ gewürdigt. Mit der treuhänderischen Verwaltung ist folgendes gemeint: Über eine vertragliche Vereinbarung überträgt ein Betriebseigentümer die Bewirtschaftung seines Betriebsvermögens an eine juristische oder natürliche Person, die im Management befähigter ist und die Unternehmungsrisiken tragen kann. Dieser Auftragnehmer erhält ein Entgelt. Das Verhältnis zwischen dem Betriebseigentümer und dem Auftragnehmer sowie dessen Pflichten, Rechte und Interessen sind im Vertrag klar definiert. Ziel dieses Modells ist der Erhalt und die Vermehrung des Betriebsvermögens.

- Einführung eines Aktiensystems, Entfaltung der positiven Funktion des Kapitalmarktes für die strategische Umstrukturierung des SEU. Der Aufbau eines modernen Betriebssystems und die Realisierung positiver Skaleneffekte machen es erforderlich, den großen Teil der SEU entsprechend eines Aktiensystems zu reformieren. Die Entwicklung des Aktiensystems setzt einen Kapitalmarkt in größerem Umfang voraus. Bei der strukturellen Regulierung soll der Entfaltung der positiven Funktion des Kapitalmarktes große Aufmerksamkeit geschenkt werden. Angesichts der realen Situation des Kapitalmarktes in unserem Land soll beachtet werden, dass die Umwandlung in ein Aktiensystem, obzwar sie einen unumkehrbaren Trend darstellt, nicht überstürzt in einer massenhaften Aktion geschehen soll. Es soll verhindert werden, dass 
nur an der Form, nicht an den Inhalten etwas geändert wird, nach dem Motto: nur das Wasser wird ausgewechselt, die Medizin bleibt im Topf. Letztendlich heisst es zu verhindern, dass die Reform als Köder zum bloßen Gelderwerb mißbraucht wird. Außerdem sollen $\mathrm{GmbHs}$ und genossenschaftliche Aktiengesellschaften die Hauptformen des Aktiensystems darstellen. Die Einrichtung von Aktiengesellschaften soll hauptsächlich bei großen und mittelgroßen SEU geschehen, die zukunftsträchtig sind. Zudem soll die Menge der an die Gesellschaft verkauften Aktien und das Tempo der Börsennotierung strikt kontrolliert werden. Dadurch sollen die begrenzten Ersparnisse der Gesellschaft an der richtigen Stelle verwendet werden. Zusammengefaßt soll das Wachstum der Börsennotierung und des Gesamtkapitalvolumens, das die Aktiengesellschaften aus der Gesellschaft sammeln, niedriger sein als die Wachstumsraten des Bruttosozialprodukts und des Volkseinkommens. Dadurch sollen die Stabilität des Bankwesens und die Interessen der Investoren geschützt werden. Es soll auch verhindert werden, dass „der See ausgetrocknet wird, um Fische zu fangen“. Der Vorteil des Aktiensystems besteht in der Funktion, Kapital zu konzentrieren, und in der Mobilität des Eigentumsrechts. Beides wird heutzutage besonders benötigt und muss über verschiedene Finanzierungsinstrumente und -wege des Kapitalmarktes realisiert werden. Die optimale Form zur Schaffung großer Unternehmen und Unternehmensgruppen ist die Verbindung von zwei starken Unternehmen. Im Ausland wird dies normalerweise über einen Aktientausch zwischen den Unternehmen geregelt. Daher benötigt die Entwicklung des Aktiensystems in erster Linie einen breit angelegten Kapitalmarkt, inklusive eines Aktienmarktes. Der Kapitalmarkt unseres Landes befindet sich heute noch in einem Anfangsstadium mit einem niedrigen Grad an Jurisdiktion und Standardisierung. Er ist noch weit entfernt von den Anforderungen, die die Entwicklung der Marktwirtschaft und die strategische Umstrukturierung der SEU stellen. Die Entwicklung des Kapitalmarktes soll daher tatkräftig gefördert werden.

- Die Möglichkeit von Konkursen. Unternehmen, die mit Verlusten arbeiten und langfristig keine Hoffnung auf Umwandlung der Verluste in Gewinne haben, sollen Konkurs anmelden. Die Schulden dieser Unternehmen sollen umstrukturiert werden. Im März 1993 gab der Staatsrat bekannt, dass nur die bankrotten SEU, die wirklich geschlossen und die Beschäftigten untergebracht haben und deren Boden und Vermögen auf Auktionen verkauft worden ist, ihre Schulden einschließlich Zinsen über den Reservefonds der Banken zur Begleichung notleidender bzw. uneinbringlicher Kredite stornieren lassen können. Diese Mitteilung führte zur weiteren Standardisierung des Konkursverfahrens. Vorher wollten manche Unternehmen den Konkurs als Mittel zur Streichung der Schulden missbrauchen. Das Ergebnis war: Die Schuldenlast war zwar nicht mehr da, jedoch das überschüssige Personal. Zudem wurde das Management nicht umgewandelt, die Ausrüstung blieb veral- 
tet und rückständig, die Produkte fanden weiterhin keinen Absatz. Nach kurzer Zeit standen diese Unternehmen vor dem zweiten Konkurs. Dies führte zum Verlust von Staatsvermögen. Das Ziel der strategischen Umstrukturierung der SEU konnte so nicht erreicht werden.

- Methoden der Schuldenumstrukturierung. Nachdem Subventionen, die in der Phase der Planwirtschaft die wichtigste Finanzierungsquelle der staatlichen Unternehmen waren, im Laufe der Reform in Kredite umgewandelt worden waren, haben manche SEU aufgrund ihrer niedrigen Effizienz hohe Schulden durch Kredite der staatlichen Geschäftsbanken angesammelt. Es gibt zwei Methoden zur Schuldenumstrukturierung: Umwandlung der Bankforderung in Aktien, damit die Banken ihre Kontrolle und Überwachung verstärken und Verkauf der Forderungen an Aktionäre, damit diese die Manager der Unternehmen kontrollieren und überwachen.

\subsection{Konzentration der Staatskontrolle auf große Staatsunternehmen}

Eine der Zielsetzungen der strategischen Umstrukturierung der SEU ist die Entwicklung der mittelgroßen und großen Unternehmen, insbesondere der äußerst großen Unternehmensgruppen, so dass diese positive Skaleneffekte erzielen. Im Jahre 1996 gab es 15.763 SEU. An der Gesamtzahl der Staatsunternehmen hatten die großen und äußerst großen SEU einen Anteil von 5,7\%. Allerdings lag ihr Anteil an Vermögen, Output, Gewinn plus Steuerzahlungen jeweils bei 83,9\%, 82,5\% und 94\%. Aus diesem Grund sollen die starken und konkurrenzfähigen großen SEU forciert entwickelt werden. Die Förderung soll sich allerdings nicht blindlings nach der Größe bzw. dem Umfang der SEU richten. Dies ergibt sich schon wegen der Besonderheit der Klassifikation der SEU in China: Manche SEU sind zwar als große und mittelgroße SEU eingestuft worden, ihre Größe aber ist von der optimalen Wirtschaftsgröße weit entfernt. Daher haben diese SEU im Vergleich zu den mittelgroßen und kleinen SEU keine merklichen Wettbewerbsvorteile auf dem Markt. Die Gesamtperformance der großen SEU ist zwar besser, dazu tragen jedoch die wenigen herausragenden, guten SEU wie das Daqing-Erdölfeld, die Yuxi-Zigarettenfabrik, das Baogang-Eisen- und Stahlkombinat, Haier, Changhong u.a. bei. Unter den großen SEU gibt es in Wirklichkeit viele, die seit Jahren mit Verlusten arbeiten und keine Aussicht auf Verbesserung haben. Diese SEU sollen freigegeben werden (mit der Ausnahme von SEU, die für Land und Volk lebenswichtig sind). Aus diesem Grunde sind wir der Meinung, dass die Konzentration auf die großen Unternehmen auch die mittelgroßen und kleinen SEU berücksichtigen soll, die zukunftsträchtig sind, eine hohe Effizienz und ein gutes Management haben und sich in einer schnellen Entwicklung befinden. Diese SEU haben die besten Aussichten, große Unternehmen zu werden. 
In der heutigen Zeit, wo das staatliche Vermögen gestaffelt von verschiedenen Regierungsebenen verwaltet wird, können diese auch verschiedene Schwerpunkte setzen: Der Zentralstaat und die Provinzebenen sollen sich auf wenige große und besonders große Unternehmen konzentrieren, die ausgeprägte Wettbewerbsvorteile haben. Die Lokal- und Kreisebenen können anhand der Lage und Größe der Unternehmen ihre eigenen Schwerpunkte für die Konzentration setzen. Hier können auch mittelgroße und kleine Unternehmen berücksichtigt werden, die Wettbewerbsvorteile haben. Auf der anderen Seite soll man bei der Freigabe kleiner Unternehmen auch diejenigen großen Unternehmen heranziehen, die keine Zukunft haben, deren Management auf niedrigem Niveau arbeitet, die nicht konkurrenzfähig sind, seit Jahren in Verlusten stehen (mit der Ausnahme von politisch bedingten Verlusten) und keine Hoffnung auf Umwandlung der Verluste in Gewinne haben. Bei der Konzentration auf große und der Freigabe der kleinen SEU soll also im Grunde das Ziel erreicht werden, wettbewerbsfähige Unternehmen auszuwählen und zu fördern, nicht wettbewerbsfähige ausscheiden zu lassen.

\section{Klassifikation der Staatsunternehmen}

Aufgrund dieser Überlegungen zur Umstrukturierung der Industrie können wir die SEU in solche Unternehmen einteilen, die dem Wettbewerb ausgesetzt, und solche, die ihm nicht ausgesetzt sind. Die letzteren SEU wiederum können in zwei Gruppen unterteilt werden: in diejenigen, die Güter und Dienstleistungen für die Öffentlichkeit bereitstellen; und in diejenigen, die sich in den Basisindustrien befinden und Monopolunternehmen sind. Die Umstrukturierung der SEU soll sich an dieser Klassifikation orientieren.

\section{1. "Der Staat besitzt, der Staat bewirtschaftet" - Unternehmen, die Güter und Dienstleistungen für die Öffentlichkeit bereitstellen}

Die öffentlichen Güter (inkl. der Dienstleistungen) können in zwei Kategorien eingeteilt werden: Erstens, de facto-öffentliche Güter, die kostenlos an die gesamte Bevölkerung geliefert werden, z.B. Landesverteidigung und öffentliche Sicherheit, Straßen, Sanitäranlagen, Stadtverschönerung, ökologische Maßnahmen. Diese Güter sollen von der Regierung bereitgestellt werden, damit die Ressourcenverteilung optimal geregelt wird. Zweitens, quasiöffentliche Güter, die gegen bestimmte Gebühren den Verbrauchern geliefert werden, z.B. staatliche Schulen, Krankenhäuser, kulturelle Einrichtungen, fließendes Wasser und Gas für die Städte, Post und Telekommunikation, Medien und Rundfunk, Eisenbahnwesen, Staatsbanken.

Es besteht grundsätzlich Einigkeit darüber, dass die Einheiten, die de factoöffentliche Güter bereitstellen, von der Regierung direkt kontrolliert und verwaltet werden sollen. Wenn dagegen die SEU, die quasi-öffentlich Güter 
liefern, nach den Anforderungen des Marktes umstrukturiert werden, dann bringt es mehr Nachteile als Vorteile mit sich. Denn wenn diese Unternehmen nur den Kräften des Marktes überlassen werden, können sie nicht nur ihre Monopolstellung missbrauchen und den Verbrauchern Schaden zufügen, auch die Marktmechanismen greifen dann nicht mehr und die optimale Ressourcenverteilung wird nicht realisiert. Deshalb soll die Regierung nicht nur das Eigentumsrecht am Gesamt- bzw. größten Teil des Vermögens dieser Unternehmen besitzen, sondern diese Unternehmen auch direkt bewirtschaften, wobei die Regierung nach dem realen Bedarf der Gesellschaft das jährliche Investitionsvolumen festlegt und die Investitionsprojekte kontrolliert. Weiterhin ist die Erzielung von Gewinn nicht das Ziel der Bewirtschaftung. Die Regierung beauftragt die Verantwortlichen der Unternehmen; sie legt die Aufgaben und Ziele für die Bewirtschaftung fest und setzt eine direkte Planung um; die Finanzen der Unternehmen werden in den Staatshaushalt integriert, Einnahmen und Ausgaben erfolgen zentral, die Investitionen der Unternehmen werden durch Subventionen der zentralen Finanzbehörden bereitgestellt. Die Regierung legt das Lohnniveau der Beschäftigten fest, und die Unternehmen haben keine Macht, dies zu durchbrechen.

- Die Regierung autorisiert nur ein oder wenige Unternehmen für bestimmte Branchen, die damit monopolisiert sind. Auf diese Weise sollen Größenvorteile gesichert werden. Der Eintritt anderer Unternehmen soll kontrolliert werden, um Ressourcenverschwendung durch übermäßige Konkurrenz zu vermeiden. Zugleich soll die Möglichkeit zum Austritt der bereits am Markt agierenden Unternehmen strikt eingeschränkt werden.

- Aufgrund der Monopolstellung der Unternehmen, die quasi-öffentliche Güter liefern, sollen diese Unternehmen kein Recht haben, die Preise selbst festzulegen. Damit soll vermieden werden, dass die Unternehmen den Interessen der Verbraucher durch übermäßig hohe Preise schaden. Die Regierung legt die Preise fest, und zwar anhand der Grenzkosten der Güter, eines ordentlichen Gewinniveaus und der Zahlungsfähigkeit der Verbraucher. Wenn die Preise aufgrund der Verfolgung gesellschaftlicher Ziele unter den Kosten liegen und die Unternehmen somit politisch bedingte Verluste erleiden, sollen diese Verluste subventioniert werden.

\section{2. "Der Staat besitzt, der Staat kontrolliert" - die monopolisierten Unternehmen}

Die meisten Branchen weisen Größenvorteile auf. In manchen Branchen sind sie besonders ausgeprägt, z.B. im Automobilbau, Schwermaschinenbau, in der Stahlindustrie, elektrotechnischen Industrie. Da der Bedarf begrenzt ist, können in solchen Branchen wenige Anbieter, manchmal sogar ein einziger Anbieter, den Markt beliefern und die gesamte Nachfrage befriedigen. Ande- 
re Anbieter können nicht in diesen Markt einsteigen. Dies führt zum sogenannten natürlichen Monopol. Die Regierungen vieler Länder greifen in verschiedenen Formen in die natürlich monopolisierten Branchen ein und kontrollieren sie. Heutzutage werden solche Branchen in den meisten Ländern mit Ausnahme der USA in unterschiedlichem Ausmaß von SEU bewirtschaftet.

Wenn China als ein Entwicklungsland seine Basisindustrien angemessen entwickeln will, muss die Regierung, objektiv gesehen, eine aktive Rolle übernehmen. Auf die Monopol-SEU soll das Modell angewendet werden, nach dem der Staat das Eigentumsrecht über das Unternehmensvermögen besitzt und die Kontrolle kraft seines Aktienbesitzes ausübt. Selbst die Monopolunternehmen, welche die Voraussetzungen für die Reform nach dem Aktiensystem erfüllen, sollen in 100\%-staatseigene Unternehmen umgewandelt werden, bzw. der Staat soll den Hauptanteil der Aktien halten. Das Modell „der Staat besitzt, der Staat kontrolliert" unterscheidet sich vom Modell „der Staat besitzt, der Staat bewirtschaftet" dadurch, dass bei jenem die Regierung die Unternehmen nicht mehr direkt bewirtschaftet. Hier wird eine größere Autonomie auf vertraglicher Basis auf die Unternehmen übertragen. Die Regierung, die in der Funktion des Eigentümers handelt, berücksichtigt bei der Vertragsvereinbarung mit den Betriebsleitern sowohl die gesellschaftlichen als auch die Gewinnziele. Sie kann die Monopolunternehmen direkt oder indirekt kontrollieren.

Für das Funktionieren des Modells „der Staat besitzt, der Staat kontrolliert“ muss das Verwaltungssystem für das staatliche Vermögen vervollständigt werden. Normalerweise hält die Regierung über $50 \%$ des Vermögens an den Monopolunternehmen. Sie hält einen höheren Anteil in einigen ausgewählten Branchen und Sektoren bzw. besitzt dieses zu 100\%. Was die Unternehmen betrifft, die nach dem Aktiensystem reformiert werden sollen, so können staatliche Gesellschaften zur Vermögensverwaltung das Eigentumsrecht des Staats vertreten; sie halten in seinem Auftrag die Aktien. Unternehmen, die nicht nach dem Aktiensystem reformiert werden, werden von den fachlichen Instanzen verwaltet. Die Regierung entsendet ihre Vertreter in den Vorstand der Unternehmen und übt somit die Funktion des Eigentümers aus; zudem entsendet sie zur Kontrolle des staatlichen Vermögens Wirtschaftsprüfer in die Unternehmen. Aufgrund der wichtigen Stellung der Basisindustrien müssen die MonopolSEU nicht nur Gewinnziele verfolgen und sich um Werterhalt und -zuwachs des staatlichen Vermögens bemühen, sie müssen auch bestimmte gesellschaftliche Ziele verwirklichen. Die Regierung als Eigentümerin soll ein Indexsystem für die Evaluation der Betriebsleistungen aufstellen. Die Rechte und Pflichten, Kompetenzen und Interessen von Regierung und Unternehmen werden über Vertrag, bzw. Plansoll definiert.

Die Regierung kontrolliert die Investitionstätigkeiten der Monopol-SEU. Die betreffenden Instanzen sollen die Investitionsentscheidung, das Investitionsvolumen, den Return on Investment und die Herkunft der Investitionsmittel 
überwachen. Dies soll die Übereinstimmung zwischen dem Angebot der Basisindustrien und dem Bedarf der Gesellschaft gewährleisten.

\subsection{Die Umwandlung der dem Wettbewerb ausgesetzten SEU in Kapitalgesellschaften}

Die im Wettbewerb stehenden SEU sind solche, die vom Staat gegründet und finanziert werden, für die im großen und ganzen keine Marktzutrittsund Marktaustrittsbarrieren existieren, deren Produkte homogen sind, die einer starken Konkurrenz ausgesetzt sind und die Gewinnziele verfolgen. Diese Unternehmen sind hauptsächlich in der verarbeitenden Industrie, dem Baugewerbe, dem Handel und den Dienstleistungen konzentriert. Um das sozialistische Marktwirtschaftssystem aufzubauen, müssen diese Unternehmen sich vollständig auf den Markt einlassen. Deshalb sind die meisten dieser SEU geeignet, zu modernen Unternehmen umgewandelt zu werden, die ein klar definiertes Eigentumsrecht, klar definierte Rechte, Verantwortungen und Kompetenzen, eine Trennung zwischen der Regierungsverwaltung und der Unternehmensleitung und eine wissenschaftliche Verwaltung haben. Je nach Situation können die dem Wettbewerb ausgesetzten SEU in Aktiengesellschaften oder Gesellschaften mit beschränkter Haftung umgewandelt werden. Die Aktiengesellschaften, welche die Voraussetzungen erfüllen, sollen zu börsennotierten Unternehmen weiterentwickelt werden. Der Kern der Bildung von Kapitalgesellschaften ist die Etablierung einer eigenständigen Rechtsform. Damit sollen die Betriebe eigene Rechtspersönlichkeiten und Marktsubjekte werden, die autonom agieren und Eigenverantwortung für Gewinn und Verlust tragen. Erste Schritte der Reform sind daher zum einen die Trennung der Kompetenzen von Staat und Unternehmen, indem der Staat beispielsweise die staatlichen Gesellschaften zur Vermögensverwaltung ermächtigt, das Eigentumsrecht auszuüben. Jene wiederum können über Abkommen das Kontrollrecht, zu dem der Staat als Inhaber der Aktien befugt ist, auf Holdinggesellschaften übertragen. Letztere fungieren als Aktionäre. Zum anderen geht es um die Etablierung einer eigenständigen Rechtsform der Unternehmen. Als juristische Person besitzt das Unternehmen das Vermögensrecht. Die Aktionäre oder Gesellschafter haften für die Unternehmensschulden mit dem Einsatz der von ihnen gekauften Aktien.

Nach der Umwandlung in Kapitalgesellschaften gibt es neben den oben genannten Holdinggesellschaften noch eine Reihe institutioneller Investoren wie z.B. Fonds, Versicherungen, Finanzinstitute, Treuhandanstalten. Des weiteren hat die Herausbildung des Kapitalmarktes Voraussetzungen für Aktienbeteiligungen der Bevölkerung und ausländische Investoren geschaffen. Diese Aktienstruktur wird nicht strikt reguliert. Wenn die Aktionäre unzufrieden sind mit den Entscheidungen auf der Aktionärsversammlung oder wenn sie 
die langfristige Amortisationsperiode der Investition nicht tragen können, können sie ihre Aktien verkaufen.

\section{Die strategische Restrukturierung der Staatsunternehmen}

\subsection{Restrukturierung der Eigentumsstruktur der Staatsunternehmen}

Die Anpassung der Eigentumsstruktur der SEU ist der Hauptweg der Umstrukturierung der SEU. Durch die Umstrukturierung des Eigentumsrechts sollen sich die staatseigenen Vermögenswerte von Industrien, die in normalem Wettbewerb stehen, auf monopolisierte, gemeinnützige und Schlüsselindustrien konzentrieren. Gleichzeitig sollen bei Staatsunternehmen die großen SEU im Vordergrund stehen.

\section{Diversifikation des Eigentums bei Staatsunternehmen}

Der jetzige Zustand wird dadurch charakterisiert, dass die Eigentumsrechte an den staatseigenen Vermögenswerten nicht klar definiert sind. Dadurch verzögert sich immer wieder die Etablierung effektiver Strukturen in den Unternehmen. Die SEU, die zu 100\% staatseigen sind, sollen in Unternehmen umgewandelt werden, in denen der Staat die Kontrolle durch eine Mehrheitsbeteiligung behält. Nichtstaatliche Aktionäre (nichtstaatliche juristische und natürliche Personen) sollen zugelassen werden. Zugleich sollen die Forderungen, die die Bevölkerung über Finanzintermediäre (Investmentfonds etc.) gegen die SEU haben, in Aktien umgewandelt werden. Außerdem sollen die zu 100\% staatseigenen SEU nicht mehr nur einen einzigen staatlichen Aktionär, sondern mehrere staatliche juristische Personen als Aktionäre haben. Die Trennung der Regierungsverwaltung von der Unternehmensführung kann so umgesetzt werden, dass die Unternehmen die direkte Kontrolle durch die Verwaltungsbehörden abschütteln können und der Staat von der unbeschränkten Haftung für die Unternehmen befreit wird.

\section{Optimierung der Ressourcenallokation der Staatsunternehmen durch Flexibilität des Eigentumsrechts}

Im wesentlichen bedeutet die strategische Umstrukturierung „Systemänderung“, „Basisveränderung“ und „Vermögenswechsel“. „Systemänderung“ bedeutet Aufbau des Systems der sozialistischen Marktwirtschaft einschließlich der Erneuerung des Unternehmenssystems; „Basisveränderung“ bedeutet Umstrukturierung der Unternehmen auf der Mikroebene; „Vermögenswechsel“ bedeutet Realisierung optimaler Kapitalallokation auf der Basis der Umgruppierung der staatseigenen Vermögenswerte. Die Umstrukturierung muss mit der Regulierung der Vermögensbestände beginnen, wobei die Umschichtung der staatseigenen Vermögenswerte der kritische Punkt ist. 
Die Vermögensumschichtung ist wichtig für die Optimierung der Produktionsfaktoren, denn sie werden durch den Prozess der Vermögensumschichtung aufgeteilt und neu zusammengeführt. Die Restrukturierung des Vermögens orientiert sich an der Gewinnmaximierung. Es gibt viele Formen für die Vermögensumgruppierung, z.B. Umwandlung in Kapitalgesellschaften, Fusionen, Akquisitionen, Leasing, treuhänderische Verwaltung, Neustrukturierung des Vermögens der Unternehmensgruppen, Aufteilung von Unternehmen, Konkurse usw. Die Umwandlung in Kapitalgesellschaften, Fusionen und Konkurse sind die Hauptmittel. Die Umwandlung in Aktiengesellschaften ist der einfachste Weg für die Vermögensumschichtung. Fusionen und Akquisitionen verursachen z. Z. noch bestimmte Schwierigkeiten, sind aber die effektivsten Wege. Die beiden zuletzt genannten Methoden sollen durch die Vervollkommnung des Kapitalmarktes und flankierende Reformmaßnahmen (z.B. die Etablierung eines Systems der sozialen Sicherung) aktiv gefördert werden. Konkurse müssen trotz vieler Hindernisse und Schwierigkeiten strikt umgesetzt werden. Vermögensumschichtung ist ein Oberbegriff, der folgendes umfaßt: Umschichtung des Portfolios, der Schulden, des Eigentumsrechts, der Beschäftigten und des Managements. Allerdings hat die Wirtschaftsentwicklung Chinas seit vielen Jahren blindlings nach mengenmäßigem Wachstum gestrebt, wobei die Verbesserung der Qualität vernachlässigt worden ist. Dabei ist eine große Menge an Vermögen entstanden, zugleich jedoch auch eine Menge unprofitablen Vermögens. Deshalb muss die Struktur der staatlichen Vermögenswerte umstrukturiert und optimiert werden. Das Wachstumsmodell, das in der Vergangenheit in erster Linie auf quantitative Expansion und nicht auf Effizienz und Qualität abzielte, muß verändert werden, nur dann ist ein „Wirtschaftswunder“ möglich.

Weiterhin sind die Unternehmen unseres Landes im allgemeinen klein und können den Anforderungen an Größenvorteile nicht gerecht werden. Im Vergleich zu den 500 größten Unternehmen auf der Welt haben sie nicht nur höhere Produktionskosten, ein niedrigeres technologisches Niveau und eine schlechtere Produktqualität, sondern auch ein ineffizienteres Management. Das Gefälle in diesen Punkten ist sehr stark ausgeprägt. Da ausländisches Kapital zunehmend nach China eingeführt wird und der chinesische Markt immer internationaler wird, sind viele chinesische Unternehmen mit einer existentiellen Krise konfrontiert. In der gegenwärtigen wirtschaftlichen Situation ist es nicht möglich, ausreichende Größenvorteile durch die Erhöhung von Investitionen zu realisieren. Steigende Skalenerträge müssen vielmehr durch eine Vermögensumschichtung erreicht werden. Dies kann dadurch geschehen, dass die Vermögenswerte von nichtwettbewerbsfähigen Unternehmen in die Vermögen von wettbewerbsfähigen Unternehmen einfließen. Durch einen solchen Prozess wird die Kapitalkonzentration beschleunigt, die Unternehmen werden vergrößert und die Wettbewerbsstärke wird erhöht. 
Nur so kann vermieden werden, dass chinesische Unternehmen von ausländischen Unternehmen geschlagen und geschluckt werden.

Ein weiterer Misstand, nämlich die Durchführung von gleichgearteten Projekten im Rahmen kleiner Unternehmen, ist in China ausgeprägt. Dies kann nur eliminiert werden, wenn die Entwicklung von großen Unternehmensgruppen vorangetrieben wird. Die Praxis zeigt, dass die regionen- und branchenübergreifenden Fusionen, die hauptsächlich von großen Unternehmensgruppen vorgenommen werden, die verwaltungsbedingte Teilung, die durch die Existenz von Provinzen als Eigentümer von SEU entsteht, überwinden kann. Dies ermöglicht eine Vermögensumschichtung in größerem Umfang. Das Problem, dass jede Provinz eine ähnliche regionale industrielle Struktur hat und es dadurch zu ineffizienten Verdoppelungen kommt, kann so besser gelöst werden.

Die Vermögensumschichtung ist die Voraussetzung für den Aufbau eines modernen Unternehmenssektors, der durch klare Rechte und Pflichten der Eigentümer sowie des Managements und einer modernen Verwaltung definiert ist. Weitere Probleme müssen auf dem Weg zu einem modernen Unternehmenssektor gelöst werden. Nur wenn die Probleme des überschüssigen Personals, der hohen sozialen Belastung und der notleidenden Schulden der SEU gelöst werden, können die Unternehmen unter modernen Bedingungen operieren. Für eine effiziente Vermögensumschichtung existieren noch viele Hindernisse. Zur Zeit sind die SEU lokal aufgeteilt und werden von bestimmten Behörden verwaltet. Dies behindert die regionen- und sektorenübergreifende Vermögensumschichtung in hohem Maße. Außerdem basiert das heutige Steuersystem immer noch auf dem traditionellen System. Obwohl das Steuersystem nach 1994 einer großen Reform unterzogen worden ist, bezahlen die Unternehmen ihre Körperschaftssteuer noch nach ihrer verwaltungsmäßigen Zugehörigkeit. Das bedeutet, dass die regionen- und branchenübergreifende Vermögensumgruppierung zur Folge haben könnte, dass sich die Empfänger der Körperschaftssteuer ändern. Daher kann es sein, dass bestimmte Verwaltungsbehörden bei Vermögensumschichtungen ihre ursprünglichen Einnahmen verlieren und sich somit ihr Interesse an einer Restrukturierung der Unternehmen reduziert.

\subsection{Probleme von Unternehmensgruppen}

Einige Unternehmensgruppen haben unterstützt von Regierungsinstanzen ihre Absicht bekundet, innerhalb weniger Jahre in die Gruppe der 500 größten Unternehmen der Welt aufzurücken. An manchen Orten hat man daher gegen wirtschaftliche Gesetzmäßigkeiten und den Willen einzelner Unternehmen Großunternehmen zusammengebastelt, wobei es nur darum geht, möglichst schnell zu großen Unternehmensgruppen zu kommen. Die Expansion kann auf zwei Wegen erfolgen. Erstens intern durch Kapitalakkumulation, 
basierend auf Wettbewerbsvorteilen in Technologie, Finanzausstattung und Management. Zweitens extern über Akquisitionen und Fusionen, d.h., man benutzt andere Unternehmen zur Vergrößerung. Zur Zeit halten nicht wenige Unternehmensgruppen und Regierungsinstanzen den ersten Weg für zu langsam. Manche Unternehmensdirektoren haben deutlich gesagt, dass ihre Unternehmensgruppen nach dem jetzigen Tempo es in drei, fünf oder auch zehn und mehr Jahren nicht schaffen würden, sich in die 500 größten Unternehmen der Welt einzureihen. Deshalb betonen sie den zweiten Weg als geeignetes Mittel der Expansion. Manche Unternehmen gehen allerdings auf Expansionskurs, indem sie mit Unternehmen fusionieren, die keine Wettbewerbsvorteile haben und manche lokale Regierungen wandeln flächendeckend eine ganze Branche in ein einziges großes Unternehmen um.

In der Tat können Umsatz und Vermögensvolumen mit solchen Expansionsmethoden in kurzer Zeit vergrößert werden. Die Qualität und die Organisationsstruktur solcher Unternehmensgruppen, das Niveau von Forschung und Entwicklung, Management usw. hat sich damit aber noch nicht wesentlich geändert. Oftmals werden solche unüberlegte Zusammenschlüsse gesunde Unternehmen in ihrer Existenz gefährden. Das Ziel der strategischen Restrukturierung des Unternehmenssektors kann so nicht erreicht werden.

Oft läßt man sich von dem günstigen Preis des Unternehmens, das man übernehmen möchte, verleiten. Sehr viele Unternehmensgruppen erleben durch die Umstrukturierung nach Akquisition und Fusion eine daraus resultierende schnelle Entwicklung. Damit geben sie auch zahlreichen mittelgroßen und kleinen Unternehmen neue Impulse. Zugleich haben manche Unternehmensgruppen zu einseitig die niedrigen Anfangskosten einer Expansion im Auge: Sie gründen und kaufen mittelgroße und kleine Unternehmen oder fusionieren mit ihnen, so dass sie Dutzende oder sogar hunderte Kinder, Enkel und Urenkel haben. Das Managementsystem hat sich dadurch aber nicht entsprechend verbessert. Die Managementkette der Unternehmensgruppe ist zu lang geworden, die Verwaltungskosten sind gestiegen und die Effizienz ist gesunken.

Es muss beachtet werden, dass Akquisitionen und Fusionen sehr risikoreiche Aktivitäten sind. Es mangelt in China nicht an Fällen, wo Unternehmen nach einer missglückten Akquisition oder Fusion untergingen. Es muss abgewogen werden, ob die Kosten für die Expansion hoch oder niedrig sind. Es gibt bei der aktuellen Vermögensumschichtung eine populäre Vorgehensweise, nämlich Fusionen und Akquisitionen mit Schuldenübernahme. In diesen Fällen werden die Schulden ganz oder zum größeren Teil übernommen. Die Schulden werden dann nach den einschlägigen Vorschriften der Regierung in Raten zurückgezahlt. Äußerlich gesehen haben die Unternehmensgruppen bei der Akquisition und Fusion nichts bezahlt, zudem können sie danach mit den Gewinnen der übernommenen Unternehmen die Schulden bezahlen. 
Soweit betrachtet ist es eine kostenlose Expansion. Allerdings gibt es hier einige blinde Flecken. Erstens verursachen die Verhandlungen, die Unterzeichnung und Umsetzung des Vertrags Kosten - sowohl Reisekosten als auch Personalaufwand. Zweitens sind die offenen und verdeckten Kosten der einverleibten Unternehmen möglicherweise nicht berücksichtigt worden. Nach der Fusion muss die Zentrale der Unternehmensgruppe Verwaltungspersonal in die übernommenen Unternehmen entsenden, die Organisationsstruktur umgestalten, Kapital zufließen lassen oder neue Technologien einführen. All dies kostet Geld. Drittens wird nur der kurzfristige Gewinn gesehen. Langfristig gesehen ist es aber sehr schwer einzuschätzen, ob übernommene Unternehmen auf dem Markt Wettbewerbsvorteile erringen können. Unter Umständen wird viel Kapital benötigt, um diese Unternehmen mittel- und langfristig zu erhalten. Wenn die übernommenen Unternehmen nicht konkurrenzfähig sind, dann werden sie zur Last. Viertens werden nur die direkten Kosten kalkuliert, nicht aber die Opportunitätskosten.

Es wird außerdem zu sehr nach Diversifikation gestrebt. Im Augenblick akquirieren und fusionieren nicht nur viele Unternehmensgruppen in der eigenen Branche, sie steigen auch in andere Branchen ein, um schnell expandieren zu können. Viele Unternehmensgruppen kündigen an, eigene Schlüsselindustrien zu entwickeln. Sie glauben, so die Unternehmensrisiken streuen und eine stabile Entwicklung sichern zu können. Wir sind der Meinung, dass diese Diversifikation die Unternehmensrisiken vermehren könnte. Zugegebenermaßen ist die Diversifikation wichtig, aber sie reduziert nicht unbedingt die Risiken. Äußerlich kann es den Anschein erwecken, die Risiken reduziert zu haben, wenn man „nicht alle Eier in einen Korb legt“. In Wirklichkeit aber können die Risiken größer werden, wenn die Unternehmen sich in den Branchen, in die sie expandiert haben, nicht gut auskennen. Je höher der Diversifikationsgrad eines Unternehmens, desto zahlreicher sind die Koordinationsaktivitäten und die möglichen Fehlentscheidungen des Managements. Die Diversifikation in nichtverwandte Branchen zwingt Eigentümer und das Topmanagement, in völlig neuen Gebieten zu wirtschaften. Sie kennen die Branchen der übernommenen Unternehmen nicht gut und können somit keine weisen Entscheidungen treffen. Zugleich führen solche Akquisitionen und Fusionen zur schnellen Zunahme von Unternehmenstöchtern in verschiedensten Gebieten. Die Manager in der Zentrale haben dann keine Zeit, sich mit den neuen Produkten vertraut zu machen und Fachwissen zu erwerben. Ihr vorhandenes Wissen reicht nicht, um die Vorschläge und Leistungen der Verantwortlichen richtig zu beurteilen. Die Überforderung der Zentrale führt oft dazu, dass die Unternehmen nach solchen Fusionen keine Größenund Verbundvorteile erreichen. Die niedrige Effizienz nach der Fusion zwingt die Unternehmen oft dazu, erneut Vermögen zu veräußern, Abteilungen und ganze Einzelunternehmen zu verkaufen. 
Zusammengefaßt kann man feststellen, dass die Diversifikation nicht unbedingt die Unternehmensrisiken reduziert. Ein altes chinesisches Sprichwort sagt: „Zehn Finger des Gegners zu verletzen ist weniger als einen Finger abzuschneiden." Nur wenn das Kerngeschäft aufgebaut worden ist, die Basis sich gefestigt hat und die Größenvorteile sich entwickelt haben, wird ein Unternehmen in der Lage sein, in der Konkurrenz auf dem inländischen und ausländischen Markt zu bestehen.

Übersetzung aus dem Chinesischen von Hong-Wei Li und Siegfried Alt 